\title{
Goal-directed fluid therapy- a survey of anaesthetists in the UK, USA, Australia and New Zealand
}

\author{
Sanket Srinivasa ${ }^{1 *}$, Arman Kahokehrr, Mattias Soop ${ }^{1}$, Matthew Taylor ${ }^{3}$ and Andrew G Hill ${ }^{1}$
}

\begin{abstract}
Background: Goal-directed fluid therapy (GDFT) has been shown to reduce complications and hospital length of stay following major surgery. However, there has been no assessment regarding its use in clinical practice.

Methods: An electronic survey was administered to randomly selected anaesthetists from the United Kingdom $(U K, n=2000)$ and the United States of America (USA, $n=2000$ ), and 500 anaesthetists from Australia/New Zealand (AUS/NZ). Preferences, clinical use and attitudes towards GDFT were investigated. Results were collated to examine regional differences.
\end{abstract}

Results: The response rates from the UK $(n=708)$ and AUS/NZ $(n=180)$ were $35 \%$, and $36 \%$ respectively. The response rate from the USA was very low $(n=178 ; 9 \%)$. GDFT use was significantly more common in the UK than in AUS/NZ ( $<$ 0.01). The Oesophageal Doppler Monitor was the most preferred instrument in the UK $(n=362 ; \mathrm{h} 76 \%)$ with no clear preferences in other regions. GDFT was most commonly utilised in major abdominal surgery and for patients with significant comorbidities. The commonest reasons stated for not using GDFT were either lack of availability of monitoring tools (AUS/NZ: 57 (70\%); UK: 94 (64\%)) or a lack of experience with instruments (AUS/NZ: 43 (53\%); UK: 51 (35\%)). A subset of respondents (AUS/NZ: 22(27\%); UK: 45 (30\%)) felt GDFT provided no perceived benefit. Enthusiasm towards the use of GDFT in the absence of existing barriers was high.

Conclusion: Several hypotheses were generated regarding important differences in the use of GDFT between anaesthetists from the UK and AUS/NZ. There is significant interest in utilising GDFT in clinical practice and existing barriers should be addressed.

Keywords: Intravenous fluid, Goal-directed fluid therapy, Perioperative care, Surgery

\section{Background}

Over the last decade, there has been a renewed emphasis on research investigating perioperative fluid therapy $[1,2]$. This has been complemented by a surge in research exploring optimised perioperative care [3-5]. An increasing body of work has now highlighted the importance of physiologically-guided, individualised fluid administration in keeping with the broad principles recommended by Moore and Shires over 40 years ago [6,7]. This concept is known as goal-directed fluid therapy (GDFT).

\footnotetext{
* Correspondence: sanketsri@gmail.com

${ }^{1}$ Department of Surgery, South Auckland Clinical School, Middlemore

Hospital, University of Auckland, Auckland, New Zealand

Full list of author information is available at the end of the article
}

GDFT therapy primarily involves the administration of intravenous fluids to optimise pre-defined, patient-specific clinical proxies of tissue perfusion [7]. The individual parameters used to guide therapy are measures of cardiovascular function and vary depending on the monitoring system used [7]. In practice, GDFT involves repeated administration of small boluses of intravenous fluids- often colloids- until a certain target or plateau is reached. A baseline infusion of crystalloids is typically given.

A number of randomised trials and subsequent metaanalyses in various surgical settings have shown that GDFT confers clinical benefits such as decreased postoperative morbidity and decreased hospital length of stay as compared to traditional, liberal fluid therapy [8-14]. This is thought to be on the basis of treating

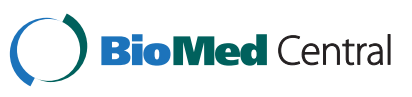


Table 1 Survey questions

Questions
Which country do you work in?
What is your place of practice? (choose as many as applicable)
What is your subspecialty interest? (up to three choices)

Options

Tertiary Hospital/District (Community) Hospital/Private practice

Abdominal Surgery/Non-abdominal general surgery/Orthopaedic surgery/Urology/ Cardiothoracic surgery/Obstetrics/Gynaecology/Vascular Surgery/Neurosurgery/ Otorhinolaryngology/Ophthalmology/Plastic Surgery/Paediatric Surgery/Trauma/ Burns

What is your current position?

Trainee/Consultant $<5$ years/Consultant $5-10$ years/Consultant $>10$ years

For adult patients having major elective surgery. . .

What is your preferred intraoperative crystalloid fluid?

What is your preferred intraoperative colloid fluid?

Normal saline/Balanced salt solution/Dextrose saline/No preference

Succinylated gelatin/Tetrastarch/Pentastarch/Hetastarch/Albumin/Nil/No preference/Other

What is your preferred intraoperative pressor? (please state type)

For adult patients undergoing major elective surgery, do you use Always/Sometimes/Never goal-directed fluid therapy?

If you use goal directed fluid therapy sometimes, when? (Choose as many as applicable)

If yes, what kind of surgery do you use goal directed fluid therapy in? (choose as many as applicable)

Major surgery/Patients with significant comorbidities/Particular operations (state type)/Depending on Instrument availability/Random/Other

Abdominal Surgery/Non-abdominal general surgery/Orthopaedic surgery/Urology/ Cardiothoracic surgery/Obstetrics/Gynaecology/Nascular Surgery/Neurosurgery/ Otorhinolaryngology/Ophthalmology/Plastic Surgery/Paediatric Surgery/Trauma/ Burns

What are your preferred tools to conduct Goal Directed Fluid ODM/PPV monitors/SW monitors/LiDCO/PAC/SvO2/PVI

Therapy? (choose up to 3)

If you never use goal directed fluid therapy, why? (choose as many as applicable)

Lack of resources/Nil advantage perceived/Too labour intensive/Unsuitable patients/Lack of experience with instruments

If existing barriers were removed (e.g. lack of resources/training), Yes/No/Undecided would you like to use Goal Directed Fluid Therapy?

ODM: Oesophageal Doppler monitor; PPV: Pulse pressure variation; SVV: Stroke volume variation; LiDCO: Lithium dilution coefficient; PAC: Pulmonary artery catheter; SvO2: Venous oxygen saturations; PVI: Pleth variability index.

occult hypovolaemia, preventing fluid overload or correcting a preoperative functional intravascular deficit early in the intraoperative phase to decrease the post-surgical inflammatory response [9-11]. The National Institute for Health Research and the Health Technology Assessment committee in the United Kingdom (UK) found in their systematic review that GDFT using the Oesophageal Doppler Monitor (ODM) provides clinical benefits in major surgery and is cost effective [15]. The National Health Service Centre for Evidence-based Purchasing also concluded that the cost of the ODM would be offset by the clinical benefits seen [16]. The use of GDFT tools is also reimbursed in the United States of America (USA) by the Center for Medicare and Medicaid Services who have stated that the use of the Oesophageal Doppler Monitor (ODM) is both "reasonable and necessary" for operative patients requiring fluid optimisation $[17,18]$. As a result, a number of guidelines from professional groups have recommended the use of GDFT in major surgery in selected cases $[19,20]$.

However, some authors have expressed reservations regarding the proposed benefits of GDFT for all patients. The criticisms include a paucity of trials within an optimised perioperative care environment and no comparison to intraoperative fluid restriction, which is also recognised as beneficial in a similar cohort of patients $[2,14,21,22]$. A recent qualitative review has also outlined several important unanswered questions in the context of GDFT in colorectal surgery [23]. Moreover, questions persist regarding the best monitoring system, the ideal intraoperative fluid

Table 2 Respondent characteristics

\begin{tabular}{llll}
\hline & AUS/NZ & UK & p-value* \\
\hline No of responders (\%) & 180 & 708 & \\
Response rate & $36 \%$ & $35 \%$ & \\
Place of practice (\%): & & & \\
\hline Tertiary Hospital District Hospital & $122(69)$ & $360(51)$ & $<0.01$ \\
Private Practice & $34(19)$ & $377(54)$ & $<0.01$ \\
& $68(38)$ & $89(13)$ & $<0.01$ \\
Experience (\%): & & & \\
\hline Trainee & $2(1)$ & $168(24)$ & $<0.01$ \\
Consultant $<\mathbf{5}$ years & $27(15)$ & $161(23)$ & 0.02 \\
Consultant $6-10$ years & $28(16)$ & $98(14)$ & 0.63 \\
Consultant $>\mathbf{1 0}$ years & & & \\
\hline
\end{tabular}

*Two-tailed Fisher's exact test. 
Table 3 Intraoperative fluid preferences

\begin{tabular}{lll}
\hline & AUS/NZ & UK \\
\hline Crystalloid (\%) & $\mathrm{n}=174$ & $\mathrm{n}=692$ \\
0.9\% Saline & $10(6)$ & $14(2)$ \\
Balanced Salt Solution & $162(93)$ & $662(96)$ \\
Colloid (\%) & & \\
\hline Succinylated gelatine & $43(24)$ & $292(42)$ \\
Tetrastarch & $30(17)$ & $98(14)$ \\
Pentastarch & $29(16)$ & $50(7)$ \\
Hetastarch & $29(16)$ & $124(18)$ \\
Albumin & $14(8)$ & 1 \\
No preference & $26(15)$ & $98(14)$ \\
Other & $8(4)$ & $29(4)$ \\
\hline
\end{tabular}

and clinical situations when GDFT is appropriate [7,23,24]. Therefore, there is clinical and academic equipoise regarding the proposed benefits or otherwise of GDFT.

For those in favour of GDFT, the translation of evidence into practice can be a significant hurdle $[25,26]$. Clinical benefits necessitate proven interventions to be suitably implemented. Selective implementation of interventions can often fail to replicate the benefits observed in trials [25]. It is thus important to assess the current practice and attitudes of clinicians and to identify barriers that may prevent the implementation of evidence-based practice $[26,27]$. Therefore, we conducted a survey of anaesthetists nationally and internationally to investigate various characteristics regarding GDFT across Australasia, the United Kingdom (UK) and the USA. Specific aims included examining the disparity in clinical uptake of GDFT, situations within which it was used and the preferred tools as well as the barriers to adoption of this technique.

\section{Methods}

Ethical approval was obtained from the Northern X Regional Ethics Committee (NTX/10/EXP/147). An electronic survey was created using a commercially available internet-based service [28]. The survey was administered electronically to 2000 randomly chosen members of the Association of Anaesthetists of Great Britain and Ireland (AAGBI), 2000 randomly chosen members of the American Society of Anesthesiology (ASA) and to 500 randomly chosen members of the Australia and New Zealand College of Anaesthetists (ANZCA) as per the conditions stipulated by each organisation. Randomisation and survey admitration was facilitated by the individual member organisations with respondents remaining anonymous to the investigators. One reminder was sent to the non-responders 4 weeks after the first invitation. The questions from the survey are presented in Table 1. Results were subsequently collated and the two-tailed Fisher's exact test was used to assess categorical outcomes. A p value of less than 0.05 was considered statistically significant.

\section{Results}

The demographic characteristics of respondents and the response rates are presented in Table 2. The response rates in the UK and AUS/NZ were 35\% and 36\% respectively. The response rate in the USA was $9 \%$, thereby limiting the validity of any deductions from these data. Amongst responders, the survey completion rate was high across all three regions (UK: 94\%; AUS/NZ: 89\%). The two commonest subspecialty interests were orthopaedic surgery (AUS/NZ: 76 (46\%); UK: $240(37 \%)$ ) and abdominal surgery (AUS/NZ: 71(43\%); UK: 300 (46\%)). Intraoperative fluid preferences are as per Table 3. The two most favoured pressors in the UK and AUS/NZ

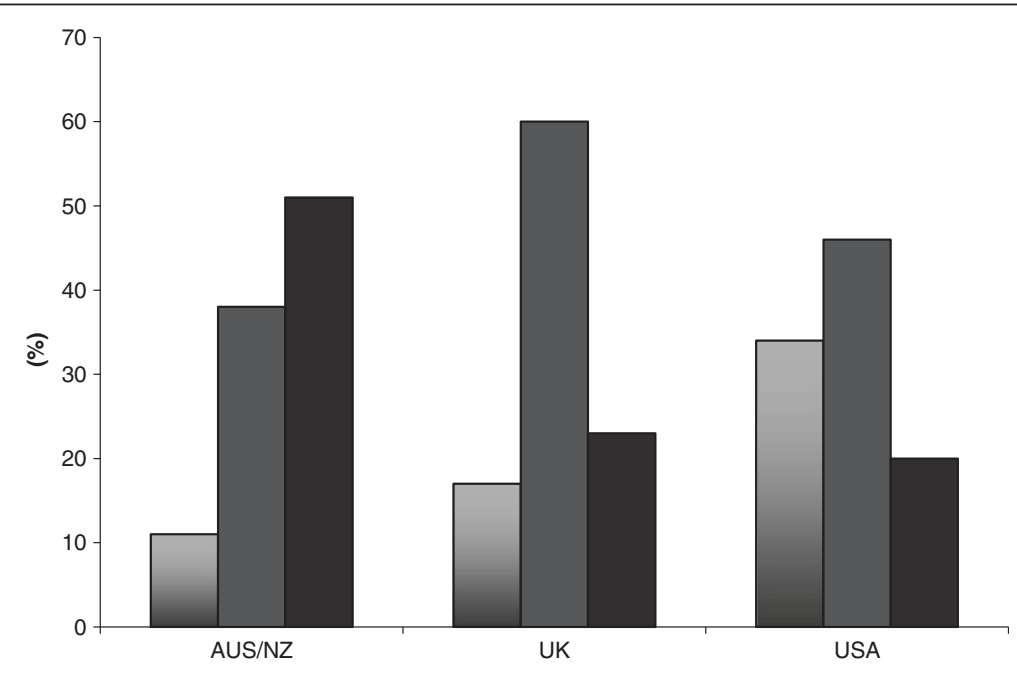

Figure 1 Do you use Goal-directed fluid therapy? (Columns from left to right respectively: Always; Sometimes; Never). Always/Sometimes: USA vs. UK, $p=0.36$; USA vs. NZ, $p<0.01$; UK vs. NZ, $p<0.01$. 
were metaraminol (UK: 281 (42\%); AUS/NZ: 102 (59\%)) and phenylephrine (UK: 142 (22\%); AUS/NZ: 36 (21\%)).

The use of GDFT is shown in Figure 1. GDFT use was significantly more common in the UK as compared to AUS/NZ ( $<<0.01)$. In AUS/NZ, GDFT was most commonly utilised in abdominal surgery $(n=60 ; 80 \%)$, followed by orthopaedic surgery $(n=27 ; 36 \%)$. In the UK, GDFT was most commonly utilised in abdominal surgery (UK: $n=428(89 \%)$ ), followed by vascular surgery (UK: $\mathrm{n}=146(30 \%)$ ).

The situations when GDFT is utilised and preferred tools to conduct GDFT are shown in Tables 4 and 5 respectively. The commonest reasons stated for not using GDFT were either lack of availability of monitoring tools (AUS/NZ: 57 (70\%); UK: 94 (64\%)) or a lack of experience with instruments (AUS/NZ:43 (53\%); UK: 51 (35\%)). A subset of respondents cited "nil advantage perceived" as the reason for not using GDFT (AUS/NZ: 22(27\%); UK: $45(30 \%))$. Enthusiasm towards GDFT in the absence of existing barriers (e.g. lack of equipment or experience) is shown in Figure 2.

The respondents from the USA indicated a preference towards GDFT in abdominal surgery $(n=97 ; 80 \%)$ with similar barriers to GDFT as described in other regions such as lack of availability of tools $(n=19 ; 59 \%)$, lack of experience $(n=13 ; 40 \%)$ and nil perceived advantage $(n=13$; $40 \%)$. The most favoured pressor in the USA was phenylephrine $(68 ; 40 \%)$, followed by ephedrine $(41 ; 24 \%)$.

\section{Discussion}

This survey of 1067 anaesthetists from the UK, AUS/NZ and the USA reveals important differences in practice with regards to intraoperative fluid therapy and GDFT specifically. The response rates were moderate or low, especially in the USA, and it is likely that the present data represents views of a self-selected group among the anaesthetists who were randomly invited to participate. Such selection could be based on strong positive or negative views on GDFT. Nevertheless, certain observations can be made and hypotheses generated regarding the interest in and barriers to GDFT in the UK and AUS/NZ. The poor response rate from the USA limits the validity of any statements regarding practice

$\begin{aligned} & \text { Table } \mathbf{4} \text { Situations when goal-directed fluid therapy is } \\
& \text { used }\end{aligned}$
\begin{tabular}{lll}
\hline Indication (\%) & AUS/NZ ( $\mathbf{n}=\mathbf{8 7})$ & UK ( $\mathbf{n}=\mathbf{5 2 3})$ \\
\hline Major Surgery & $50(69)$ & $326(73)$ \\
Significant Comorbidities & $51(70)$ & $367(83)$ \\
Specific Operations & $20(27)$ & $107(24)$ \\
Instrument availability & $19(26)$ & $125(28)$ \\
Random & 0 & $11(3)$ \\
\hline
\end{tabular}

Table 5 Preferred tools for goal-directed fluid therapy

\begin{tabular}{lll}
\hline Tools (\%) & AUS/NZ $(\mathbf{n}=\mathbf{7 8})$ & UK $(\mathbf{n}=\mathbf{5 1 9})$ \\
\hline ODM & $11(19)$ & $362(76)$ \\
PPV & $26(45)$ & $97(20)$ \\
SW & $21(36)$ & $71(15)$ \\
LiDCO & $1(2)$ & $93(20)$ \\
PAC & $17(29)$ & $35(7)$ \\
SVO2 & $12(21)$ & $88(19)$ \\
PVI & $3(5)$ & $8(2)$ \\
\hline
\end{tabular}

ODM: Oesophageal doppler monitor; PPV: Pulse pressure variation; SVV: Stroke volume variation; LiDCO: Lithium dilution coefficient; PAC: Pulmonary artery catheter; SvO2: Venous oxygen saturation; PVI: Pleth variability index.

in this region except to speculate that there is comparatively lesser interest in this topic.

The use of GDFT seems to be significantly less prevalent in AUS/NZ compared to the UK amongst respondents in this survey. The majority of the respondents were involved in major abdominal surgery and orthopaedic surgery and used GDFT in patients with significant comorbidities. The ODM is the most commonly utilised instrument in the UK with significant variation in preferences in other regions. The most significant barriers to conducting GDFT were either a lack of availability of monitoring tools or a lack of experience with instruments. Even though a proportion of respondents from all regions remain unconvinced of the benefits of GDFT, there was significant enthusiasm overall towards trialling GDFT if barriers were to be removed.

The use of GDFT was most common in the UK which may have been made possible by governmental endorsement and funding of the ODM specifically with demonstrated cost-effectiveness $[15,16]$. Nationally-driven implementation may also potentially overcome the problems associated with silo budgeting as GDFT represents an anaesthetic intervention- thereby adding cost to clinical departments of anaesthesia- to provide financial benefits for surgical services such as decreased hospital length of stay. This represents a potential blueprint for other regions to follow. For clinicians, the recently published GIFTASUP guidelines which recommend mandatory use of GDFT in major abdominal surgery may have also provided further impetus [20].

Moreover, a significant portion of the evidence supporting GDFT originates from the UK $[9,11,29]$. The recent guidelines from the European Enhanced Recovery After Surgery (ERAS) group have also shown enthusiasm towards GDFT and the ODM and, as ERAS protocols are instituted across the UK, GDFT has been integrated into practice as well $[19,30,31]$. This has likely led to the emergence of the ODM as the preferred tool to conduct GDFT in the UK. In contrast, the principles of optimised perioperative care have shown reduced penetrance in AUS/NZ with persisting scepticism regarding benefits 


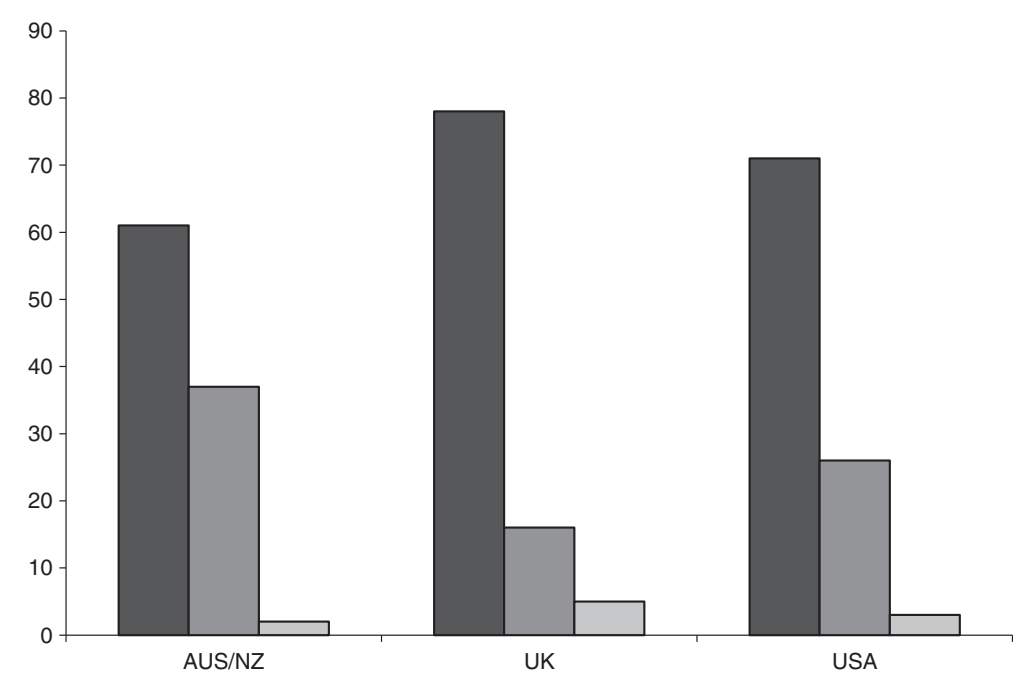

Figure 2 Would you like to use Goal-Directed Fluid Therapy? (Columns from left to right respectively: Yes/No/Undecided).

$[32,33]$. In AUS/NZ, there appears to be no clear preference with regards to tools for GDFT.

The barriers identified to the use of GDFT appear eminently solvable especially if the observed benefits from trials are replicated in clinical practice [13]. Many of the instruments used to conduct GDFT are simple to use and the learning curve for the ODM can be overcome after 12 insertions [34]. However, it should be noted that in the context of abdominal surgery, restrictive fluid regimens have also shown similar benefits to GDFT and the majority of the trials investigating GDFT have not been conducted in an environment of standardised, optimised perioperative care $[14,23]$.

A proportion of people from all the regions surveyed remain sceptical regarding the proposed benefits of GDFT. To an extent, this is justified as important questions remain unanswered, such as efficacy in settings where fluid restriction has been shown to be beneficial [21-23]. Nonetheless, it is interesting to note that in the absence of barriers, a high proportion of respondents would be willing to consider GDFT into their practice. This suggests that future randomised trials or selective clinical implementation of GDFT remain feasible and are required.

Whilst the use of intraoperative crystalloids was largely homogeneous between the regions, there were interesting differences in practice between the three regions with regards to colloid use. There are considerable differences between individual classes of colloids as well as between the individual colloid products themselves $[35,36]$. Succinylated gelatin solutions were favoured in the UK and AUS/NZ with the latter showing a wide variation in practice. This may be a reflection of the variable availability and cost of these solutions.

There are important limitations to this study which need to be considered alongside the results. The survey was designed on the basis of questions of clinical importance but was not developed with a focus group nor with redundant questions to validate its methodology. Moreover, since the survey aimed towards an overview of GDFT, specifics could not be elucidated regarding any one aspect (e.g. specific barriers). Any barriers identified were the opinion of the respondent and may not be reflective of the institution or the region in its entirety. The number of individuals to be surveyed was limited by the respective professional associations. The colleges facilitated selection of individuals with no author input allowed regarding this. The only selection criterion was to prevent administration of the survey to individuals who had previously replied to other college-led surveys to minimise responder fatigue. In the context of a lowmoderate response rate, it cannot be assumed with certainty that it is representative of the anaesthetic community in its entirety.

\section{Conclusion}

There are important differences in fluid administration and the use of GDFT between anaesthetists from the UK and AUS/NZ. The identified barriers can potentially be overcome and though some clinicians remain unconvinced, there is significant interest in utilising GDFT in clinical practice. Future directions from this work can include selective implementation of GDFT or further clinical studies.

\section{Competing interests}

The authors are currently running a randomised trial exploring Oesophageal Doppler-guided fluid therapy in colonic resections within an enhanced recovery after surgery protocol. (NCT00911391). The Oesophageal Doppler machine for this trial has been lent by Pharmaco New Zealand. All probes have been bought at regular cost and Pharmaco New Zealand has had no input into the design or conduct of the trial and will not have any influence on publication. The authors of this manuscript run an annual course on the 
principles of optimised perioperative care (http://webstarts.com/aerascourse) and have received sponsorship from Pharmaco NZ for this event. Pharmaco NZ has no input into the course content or delivery.

\section{Authors' contributions}

SS- designed the survey, analysed the data, wrote the manuscript. AKanalysed the data, edited the manuscript. MS- edited the survey, revised the manuscript. MT- conceived the idea for the project, revised the manuscript. $\mathrm{AH}$-conceived the idea for the project, designed the survey, assisted in data analysis and revised the manuscript. All authors read and approved the final manuscript.

\section{Acknowledgements}

We wish to thank Stephanie Poustie (ANZCA), Chloe Hoy (AAGBI) and Celeste Kirschner (ASA) and their respective organisations for helping to administer this survey to their members.

\section{Author details}

'Department of Surgery, South Auckland Clinical School, Middlemore Hospital, University of Auckland, Auckland, New Zealand. ${ }^{2}$ Department of Surgery, North Shore Hospital, University of Auckland, Auckland, New Zealand. ${ }^{3}$ Department of Anaesthesia, Middlemore Hospital, Auckland, New Zealand.

Received: 26 July 2012 Accepted: 7 February 2013 Published: 22 February 2013

\section{References}

1. Lobo DN, Macafee DAL, Allison SP: How perioperative fluid balance influences postoperative outcomes. Best Prac Res Clin Anaesthesiol 2006, 20(3):439-55.

2. Chappell D, Jacob M, Hofmann-Kiefer K, Conzen P, Rehm M: A rational approach to perioperative fluid management. Anesthesiology 2008 109(4):723-40.

3. Zargar-Shoshtari K, Hill AG: Optimization of perioperative care for colonic surgery: a review of the evidence. ANZ J Surg 2008, 78(1-2):13-23.

4. Zargar-Shoshtari K, Connolly AB, Israel LH, Hill AG: Fast-track surgery may reduce complications following major colonic surgery. Dis Col Rect 2008, 51(11):1633-40.

5. Kehlet $H$, Wilmore DW: Evidence-based surgical care and the evolution of fast-track surgery. Ann Surg 2008, 248(2):189-98.

6. Moore FD, Shires G: Moderation. Ann Surg 1967, 166(2):300-1

7. Bundgaard-Nielsen M, Holte $\mathrm{K}$, Secher NH, Kehlet $\mathrm{H}$ : Monitoring of perioperative fluid administration by individualized goal-directed therapy. Acta Anaesthesiol Scand 2007, 51(3):331-40.

8. Giglio MT, Marucci M, Testini M, Brienza N: Goal-directed haemodynamic therapy and gastrointestinal complications in major surgery: a metaanalysis of randomized controlled trials. Br J Anaesth 2009, 103(5):637-46.

9. Noblett SE, Snowden CP, Shenton BK, Horgan AF: Randomized clinical trial assessing the effect of Doppler-optimized fluid management on outcome after elective colorectal resection. Br J Surg 2006, 93(9):1069-76.

10. Mythen MG, Webb AR: Perioperative plasma volume expansion reduces the incidence of gut mucosal hypoperfusion during cardiac surgery. Arch Surg 1995, 130(4):423-9.

11. Sinclair S, James S, Singer M: Intraoperative intravascular volume optimisation and length of hospital stay after repair of proximal femoral fracture: randomised controlled trial. Br Med J 1997, 315(7113):909-12.

12. Lopes MR, Oliveira MA, Pereira VOS, Lemos IPB, Auler JOC Jr, Michard F: Goal-directed fluid management based on pulse pressure variation monitoring during high-risk surgery: a pilot randomized controlled trial. Crit Care 2007, 11(5):R100

13. Abbas SM, Hill AG: Systematic review of the literature for the use of oesophageal Doppler monitor for fluid replacement in major abdominal surgery. Anaesthesia 2008, 63(1):44-51.

14. Rahbari NN, Zimmermann JB, Schmidt T, Koch M, Weigand MA, Weitz J: Meta-analysis of standard, restrictive and supplemental fluid administration in colorectal surgery. Br J Surg 2009, 96(4):331-41.

15. Mowatt G, Houston G, Hernandez R, de Verteuil R, Fraser C, Cuthbertson B, Vale $L$ : Systematic review of the clinical effectiveness and costeffectiveness of oesophageal Doppler monitoring in critically ill and high-risk surgical patients. Health Technol Assess, 13(7):1-95. p iii-iv, ix-xii.
16. Evidence review: Oesophageal Doppler monitoring in patients undergoing high-risk surgery and in critically ill patients. CEP08012. NHS Purchasing and Supply Agency. 2008. Also available from: www.pasa.nhs.uk/PASAWeb/ NHSprocurement/CEP.

17. CMS Decision Memo for Ultrasound Diagnostic Procedures Administrative File CAG-00309R, Final Coverage Decision Memorandum for Ultrasound Diagnostic Procedures. 2007

18. Centers for Medicare and Medicare Services, Pub 100-03 Medicare National Coverage Determinations; Transmittal 76. 2007.

19. Lassen K, Soop M, Nygren J, Cox PBW, Hendry PO, Spies C, von Meyenfeldt MF, Fearon KCH, Revhaug A, Norderval S, Ljungqvist O, Lobo DN, Dejong $\mathrm{CHC}$ : for the enhanced recovery after surgery $\mathrm{G}$. Consensus review of optimal perioperative care in colorectal surgery: Enhanced Recovery After Surgery (ERAS) group recommendations. Arch Surg 2009, 144(10):961-969.

20. Powell-Tuck JGP, Lobo DN, Allison SP, Carlson GL, Gore M, Lewington AJ, Pearse RM, Mythen MG: British Consensus Guidelines on Intravenous Fluid Therapy for Adult Surgical Patients (GIFTASUP). 2011. www.surgicalresearch. org.uk.

21. Kehlet $H$, Bundgaard-Nielsen $M$ : Goal-directed perioperative fluid management: why, when, and how? Anesthesiology 2009, 110(3):453-5

22. Drage S, Boyd O: Perioperative goal directed haemodynamic therapy do it, bin it, or finally investigate it properly? Crit Care 2007, 11(5):170.

23. Srinivasa S, Taylor MHG, Sammour T, Kahokehr AA, Hill AG: Oesophageal Doppler-guided fluid administration in colorectal surgery: critical appraisal of published clinical trials. Acta Anaesthesiol Scand 2010, p epub ahead of press.

24. Kehlet H: Fast-track colorectal surgery. Lancet 2008, 371(9615):791-793.

25. Grol R, Grimshaw J: From best evidence to best practice: effective implementation of change in patients' care. Lancet 2003, 362(9391):1225-1230.

26. Kehlet $H$, Wilmore DW: Surgical care - how can new evidence be applied to clinical practice? Colorect Dis 2010, 12(1):2-4.

27. Davis DA, Taylor-Vaisey A: Translating guidelines into practice: a systematic review of theoretic concepts, practical experience and research evidence in the adoption of clinical practice guidelines. CMAJ 1997, 157(4):408-416.

28. SurveyMonkey: www.surveymonkey.com accessed on 12/08/10.

29. McKendry M, McGloin H, Saberi D, Caudwell L, Brady AR, Singer $M$ Randomised controlled trial assessing the impact of a nurse delivered, flow monitored protocol for optimisation of circulatory status after cardiac surgery. Br Med J 2004, 329(7460):258 [Erratum appears in BMJ. 2004 Aug 21;329(7463):438].

30. American Society of Anesthesiologists: http://www.asa-abstracts.com/ strands/asaabstracts/abstract.htm; jsessionid=07A8B5605DE9FBE92799DFD76955A714? year $=2010 \&$ index $=4 \&$ absnum $=998$ visited on 12/01/2011.

31. Roberts J, Mythen M, Horgan A: Thinking differently: working to spread enhanced recovery across England. Curr Anaesth Crit Care 2010, 21(3):137-141.

32. Kahokehr A, Robertson P, Sammour T, Soop M, Hill AG: Peri-operative care: a survey of New Zealand and Australian colorectal surgeons. Colorect Dis 2011, 13(11):1308-1313.

33. Kahokehr AA, Sammour T, Srinivasa S, Hill AG: Reducing the length of stay for patients undergoing colorectal surgery. ANZ J Surg 2010, 80(3):195.

34. Lefrant J, Bruelle P, Aya A, Saïssi G, Dauzat M, de La Coussaye J, Eledjam J: Training is required to improve the reliability of esophageal doppler to measure cardiac output in critically ill patients. Intensive Care Med 1998, 24(4):347-352.

35. Traylor RJ, Pearl RG: Crystalloid versus colloid versus colloid: all colloids are not created equal. Anesth Analg 1996, 83(2):209-12.

36. Westphal M, James MFM, Kozek-Langenecker S, Stocker R, Guidet B, Van Aken H: Hydroxyethyl starches: different products - different effects. Anesthesiology 2009, 111(1):187-202.

doi:10.1186/1471-2253-13-5

Cite this article as: Srinivasa et al:: Goal-directed fluid therapy- a survey of anaesthetists in the UK, USA, Australia and New Zealand. BMC Anesthesiology 2013 13:5. 